\title{
Analysis of a Benzamide/Cholesterol Mixture by Using TLC/FTIR Technique
}

\author{
Xiaokun Fan, ${ }^{1,2}$ Ran Guo, ${ }^{2}$ Jia-jia Shi, ${ }^{1,2}$ Haijun Wu, ${ }^{3}$ Anqi He, ${ }^{2}$ Yongju Wei, ${ }^{1}$ Cuige Liu, ${ }^{1}$ \\ Shifu Weng, ${ }^{2}$ Zhanlan Yang, ${ }^{2}$ Yizhuang $\mathrm{Xu}^{2}{ }^{2}$ Isao Noda, ${ }^{4}$ and Jinguang $\mathrm{Wu}^{2}$ \\ ${ }^{1}$ College of Chemistry and Material Science, Hebei Normal University, Shijiazhuang 050024, China \\ ${ }^{2}$ College of Chemistry and Molecular Engineering, Peking University, Beijing 100871, China \\ ${ }^{3}$ Mongolia Medical College, Inner Mongolia University for the Nationalities, Tongliao 028000, China \\ ${ }^{4}$ Department of Materials Science and Engineering, University of Delaware, Newark, DE 19716, USA
}

Correspondence should be addressed to Yizhuang Xu; xyz@pku.edu.cn

Received 6 September 2013; Accepted 22 November 2013

Academic Editor: Edyta Proniewicz

Copyright (c) 2013 Xiaokun Fan et al. This is an open access article distributed under the Creative Commons Attribution License, which permits unrestricted use, distribution, and reproduction in any medium, provided the original work is properly cited.

\begin{abstract}
We applied TLC/FTIR coupled with a mapping technique to analyze a cholesterol/benzamide mixture. Narrow-band TLC plates by using $\mathrm{AgI}$ as a stationary phase were used to separate benzamide and cholesterol. The distribution of cholesterol and benzamide spots was manifested by 3D chromatogram. Benzamide and cholesterol can be successfully separated by the narrow-band TLC plate. Moreover, characteristic bands of benzamide and cholesterol can be identified from their FTIR spectra. In addition, the FTIR spectra of the separated benzamide and cholesterol spots on the narrow band TLC plate are roughly the same as the corresponding reference IR spectra.
\end{abstract}

\section{Introduction}

Thin-layer chromatography (TLC) is one of the most versatile and widely used separation methods. The concept of TLC is simple, and samples usually require only minimal pretreatment [1-3]. TLC continues to feature a broad range of applications, such as analysis of pharmaceuticals, herbal medicines and dietary supplements, biological and clinical samples, foods and beverages, environmental pollutants, and many other chemicals. Although TLC exhibits advantages in the separation of mixtures, there is a limitation in identifying separated unknown components. In most TLC experiments, separated samples are identified by color, UV-Vis absorbance, or fluorescence signals. However, it becomes difficult to detect samples that have neither UV-Vis absorbance nor fluorescence signals. Spraying visualization reagents on TLC plate is helpful sometimes. However, the utility of visualization reagents for unknown TLC spot identification is somewhat limited, since the selected visualization reagents may not be suitable for every substance with a broad range of molecular structures present in the sample. FTIR spectroscopy $[4,5]$ is a universal tool that can identify various organic compounds or metal complexes with organic ligands, even if the separated substance lacks color, UV-Vis absorbance, or fluorescence signals. Additionally, FTIR spectrum provides abundant information relevant to functional groups of separated substances. Moreover, it might be possible to recognize unknown separated spots directly on TLC plate based on their FTIR spectra. Nowadays, the progress on FTIR spectrometer instrumentation provides additional opportunity for the development of TLC/FTIR technique. For example, the development of FTIR spectrometer with a microscope attachment makes it convenient to acquire FTIR spectra even on each tiny separated sample spot on a TLC plate. In addition, the advancement on mapping/imaging techniques enables us to obtain FTIR spectra of every microregion on a TLC plate automatically. Thus, it has become possible to perform TLC/FTIR analysis in a quick and convenient manner.

As a matter of fact, great efforts have been made to develop TLC/IR technique for about 50 years. In the 1960s, TLC/IR analysis was carried out via solvent elution transfer approach $[6,7]$. In this approach, suitable solvents were used 
to dissolve each separated sample spot so that the analytes can be transferred out of the TLC plate. After removing the solvent by vaporization, IR spectrum of the obtained sample is recorded. The procedure is tedious and timeconsuming, and the analysis is under a risk of sample loss and contamination. In 1975, Griffiths et al. [8-10] published a pioneering work on the development of in situ TLC/IR technique. This approach is quick, convenient, and free of the risk of sample loss, since FTIR spectrum is directly obtained from the separated sample spot on a TLC plate. Subsequently, Griffiths et al. [9, 10], Zuber et al. [11], Lloyd et al. [12], and White [13] adopted various spectral techniques to develop a more practical in situ TLC/IR technique. However, the biggest problem of the in situ TLC/FTIR is that traditional TLC stationary phases, such as silica and alumina, exhibit strong background absorption that can bring about significant interference to sample identification. To solve the problem, Danielson et al. proposed to use zirconium oxide that has no absorbance in mid-IR region as a new stationary phase for TLC/FTIR analysis [14, 15]. However, considerable amount of water was adsorbed on zirconium oxide and brought about severe interference in the measurement of FTIR spectra. Consequently, the problem of interference from stationary phase is not adequately addressed. Further development of the in situ TLC/FTIR technique has been mainly inhibited by the problem of strong background absorbency of stationary phase. As a matter of fact, there has been surprisingly few papers $[16,17]$ relevant to TLC/FTIR published around the world during the past decade.

To break the bottleneck of the TLC/FTIR method, we proposed to use IR-transparent, insoluble inorganic salt particles as novel stationary phases for TLC/FTIR analysis. The reason for this approach is that inorganic salt is transparent for infrared light, thereby removing the interference caused by IR absorption from stationary phase. The inorganic salt should be insoluble so that the risk of stationary phase being destroyed by mobile phase can be avoided. In addition, significant efforts have been made to control the size of the inorganic salt particles to be around $100 \mathrm{~nm}$. The reasons are as follows. (1) Decreasing the size of stationary phase particles alleviates the effect of light scattering so that the quality of the corresponding FTIR spectra can be improved. (2) As the size of the particles of stationary phase decreases, the efficiency of TLC separation is enhanced. (3) Decreasing the size of the inorganic salt particle results in increasing the specific area of the stationary phase. Thus, more analytic molecules are allowed to be adsorbed on the surface of the stationary phase. This advantage is helpful in alleviating the overloading problem and improving the sensitivity of detection of separated sample by using FTIR measurement.

Based on the above consideration, a systematic work on the TLC/FTIR analysis using inorganic salts has been conducted in our laboratory [18-20]. Our experiments showed that barium fluoride and silver iodide particles may be used as new effective stationary phases for TLC/FTIR analysis. In further experiment, we realized that the thorough removal of the adsorbed water is very important for successful TLC/FTIR analysis. Barium fluoride particles tend to retain significant amount of adsorbed water, while it is much easier to remove adsorbed water completely for silver iodide particles. Thus, we focus on the TLC/FTIR studies by using AgI particles as stationary phase.

In parallel, we have made considerable effort to develop a new approach to prepare TLC plates. In the traditional way of the preparation of TLC plate, polymeric adhesive is utilized to bind stationary phase particles together. However, polymeric adhesive has strong absorbance in IR region and brings about significant interference in the FTIR detection. To solve this problem, we develop an approach called "settlement volatilization method" to prepare TLC plates without polymeric adhesive.

Recently, we propose a new technique to prepare narrowband TLC plates [21]. The advantages of the narrowband TLC are as follows. (1) The preparation time is much reduced in comparison with that of the previous preparation of a TLC plate by using "settlement volatilization method." (2) The usage of stationary phase in narrowband TLC plates decreases by about one order of magnitude in comparison with that of traditional TLC plates. This is very important for using expensive material (e.g., AgI particles) as a stationary phase. (3) The sensitivity of detection of a separated sample spot increases significantly by using narrowband TLC plates because the area of the separated spots is significantly reduced.

The application of TLC/FTIR techniques in our previous work was limited to simply separating and identifying mixtures of pigments. We notice that analysis of colorless mixture is one of the advantages of the TLC/FTIR approach. In the present work, a narrowband TLC plate by using AgI as stationary phase was utilized to analysis a mixture of benzamide and cholesterol. In addition, FTIR microscope with a mapping technique capability was used to reveal the distribution of colorless cholesterol and benzamide band on the narrowband TLC plate.

\section{Experimental}

2.1. Reagents. All reagents are of AR grade. Ethanol, chloroform, normal heptane, benzamide, cholesterol, hydrofluoric acid, and silver nitrate were obtained from Beijing Chemical Factory. Potassium iodide was a product of Sinopharm Chemical Reagent Co., Ltd.

Silver iodide was synthesized by a reaction between silver nitrate and potassium iodide. The experimental details of the preparation of silver iodide particles can be found in our previous work [19]. The diameters of the silver iodide particles are around $100 \mathrm{~nm}$.

2.2. Apparatus. A Thermo-Fischer Nicolet iN10 MX FTIR spectrometer equipped with an IR microscope was used in the experiments.

\subsection{Procedures}

2.3.1. Preparation of Mixed Sample Solutions. $0.100 \mathrm{~g}$ benzamide was dissolved in $10.0 \mathrm{~mL}$ ethanol to prepare a $1 \mathrm{wt} \%$ benzamide solution. Similarly, $1 \%$ cholesterol solution was 
prepared by dissolving $0.100 \mathrm{~g}$ cholesterol in $10.0 \mathrm{~mL}$ chloroform.

A mixed solution sample was prepared by mixing equal volumes of $1 \%$ cholesterol solution and $1 \%$ benzamide solution.

2.4. Narrowband TLC Analysis. We prepared narrowband TLC plate to conduct the experiment. The preparation process of the narrowband TLC plates is described in detail in our previous work [21].

The sample solution was manually spotted at one end of narrowband TLC plate by using a $0.3 \mathrm{~mm}$ glass capillary in diameter. After evaporation of the solvent, the narrowband TLC plate was developed by using an ethanol/heptane mixture as a mobile phase (the volume ratio between heptane and ethanol is $9: 1$ ). The typical development time was about 20 30 minutes.

2.5. In Situ TLC-FTIR Detection. Narrowband TLC plate after separation was dried at $100^{\circ} \mathrm{C}$ in a vacuum oven to remove residual mobile phase. Then, TLC plates were examined directly by using the FTIR microscope. To reveal the distribution of the cholesterol and benzamide spots on the narrowband TLC plate, FTIR mapping technique under linear scan mode was utilized. That is, FTIR spectrum of every tiny region on TLC plate $(100 \mu \mathrm{m} \times 100 \mu \mathrm{m})$ is obtained sequentially along the direction of the diffusion of mobile phase. All the spectra were recorded under reflection mode at a resolution of $16 \mathrm{~cm}^{-1}$ and 16 scans were coadded. To improve the signal-to-noise ratio, reflect spectrum from gold mirror was used as background spectrum.

3D chromatogram that is utilized to reveal the distribution of cholesterol and benzamide spots was generated via a program written in our lab by using the software of MATLAB.

\section{Results and Discussion}

After separating cholesterol and benzamide, the photograph of narrowband TLC plate is shown in Figure 1. Since both cholesterol and benzamide are colorless substances, no separated sample spot can be observed. However, the separated cholesterol and benzamide spots can be successfully identified by using FTIR spectroscopic method. In this experiment, FT-IR spectrum of each tiny region on the narrowband TLC plate is sequentially recorded. The obtained FT-IR spectra are organized along the direction of the diffusion of mobile phase so that a $3 \mathrm{D}$ chromatogram (the first dimension is wavenumber from FTIR spectrum, the second dimension is migration distance, and the third dimension is absorbance) is constructed. The $3 \mathrm{D}$ chromatogram is manifested via a contour map mode. As shown in Figure 2, two separated spots can be clearly visualized in the 3D chromatogram. Typical FTIR spectra of the two spots, which are obtained by slicing the $3 \mathrm{D}$ chromatogram, are shown as trace 1 in Figures 3 and 4. Carbonyl band around $1670 \mathrm{~cm}^{-1}$ can be found in trace 1 of Figure 3 of the lower spot. These results suggest that the corresponding spot is benzamide spot. On the other hand, $\mathrm{OH}$ stretching band around $3400 \mathrm{~cm}^{-1}$ and $\mathrm{C}-\mathrm{H}$ stretching

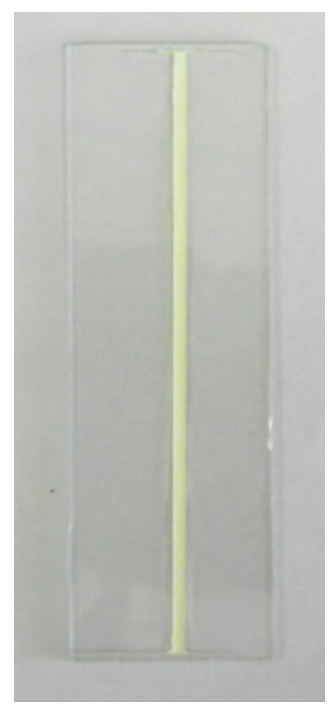

FIGURE 1: A photograph of the narrowband TLC plate after separation of cholesterol and benzamide mixtures (the width of the narrowband is $3 \mathrm{~mm}$ ).

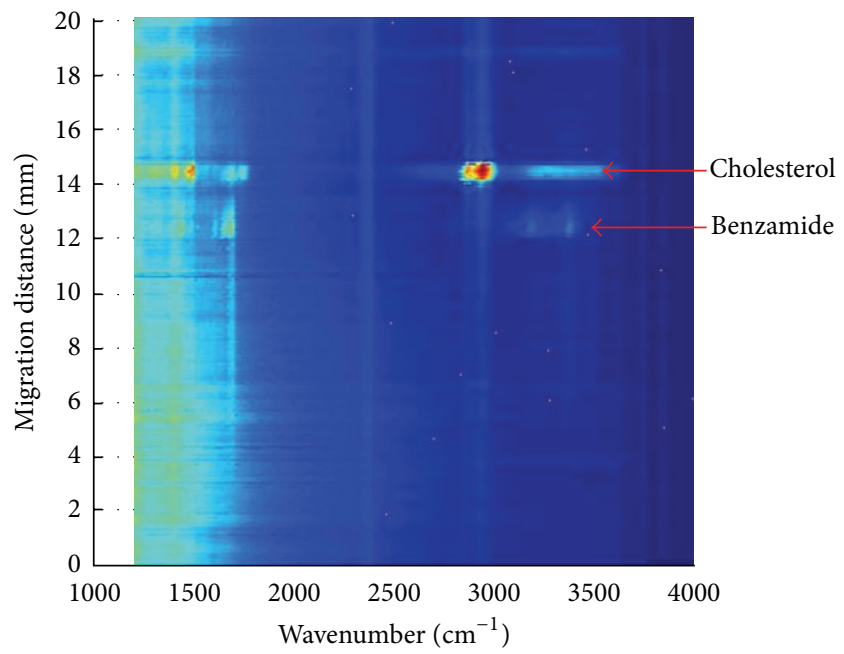

FIGURE 2: 3D chromatograph to characterize the distribution of the cholesterol and benzamide spots on a narrowband TLC plate.

band around $2900 \mathrm{~cm}^{-1}$ are observable in trace 1 of Figure 4 . This result suggests that the corresponding spot is cholesterol spot.

To confirm that the above two spots are from benzamide and cholesterol, we recorded the reference FTIR spectra of benzamide and cholesterol via the following experiments: about $0.1 \mu \mathrm{L}$ of ethanol solution containing $1 \%$ benzamide only and $0.1 \mu \mathrm{L}$ of ethanol solution containing $1 \%$ cholesterol only were manually spotted on two TLC plates. After evaporation of solvent, the TLC plate was developed by using a mixture of ethanol and normal heptane (the volume ratio of normal heptane and ethanol is $9: 1$ ) as mobile phases. Afterwards, the two plates were dried at $100^{\circ} \mathrm{C}$ in a vacuum oven to remove residual mobile phase. We use FTIR microscope mapping technique to locate benzamide and 


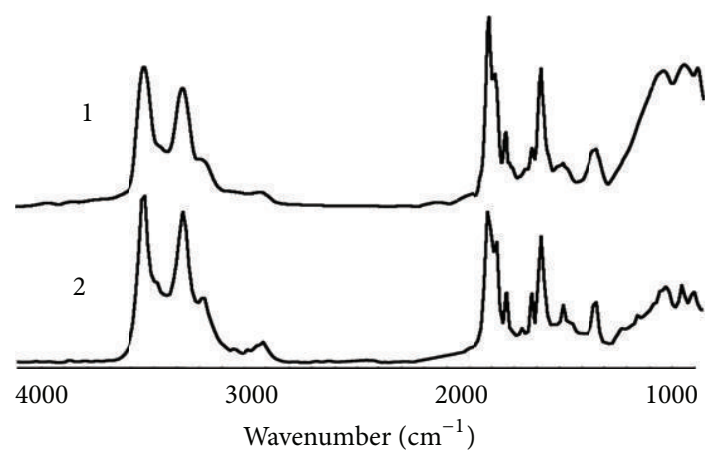

FIGURE 3: FTIR spectrum of benzamide spot (trace 1) on a narrowband TLC plate. For comparison, a reference FTIR spectrum of pure benzamide (trace 2 ) is also included.

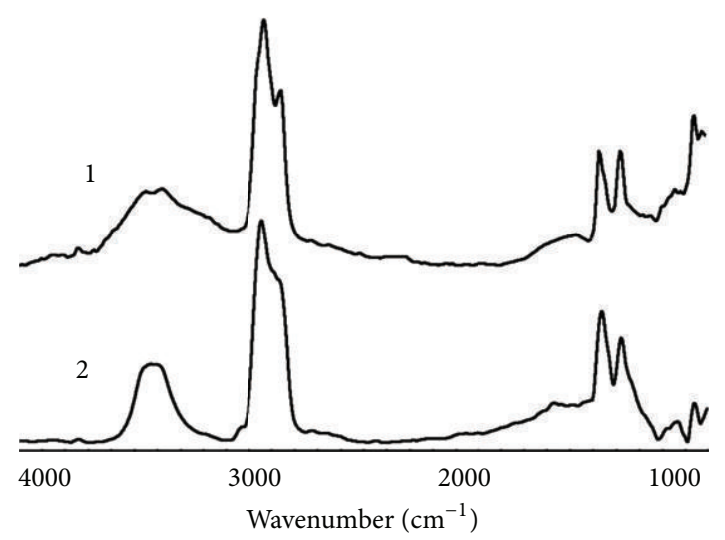

FIGURE 4: FTIR spectrum of cholesterol spot (trace 1) on a narrowband TLC plate. For comparison, a reference FTIR spectrum (trace 2) of pure cholesterol is also included.

cholesterol spots on the two narrowband TLC plates. Then, we collected FTIR spectra on the benzamide and cholesterol spots. The obtained FTIR spectra are used as reference spectra and shown as trace 2 in Figures 3 and 4 . As demonstrated in Figures 3 and 4, FTIR spectra of upper and lower spots are almost the same as the reference spectra of cholesterol and benzamide. This result confirms that the separated spots shown in Figure 2 are indeed cholesterol and benzamide.

\section{Conclusion}

In this work, we use narrowband TLC plates by using AgI as stationary phase to separate benzamide and cholesterol. Then, the distribution of benzamide and cholesterol spots was probed by using FTIR microscope with spectral mapping technique and manifested by 3D chromatogram. Experimental results demonstrate that benzamide and cholesterol can be successfully separated by the narrowband TLC plate. In addition, characteristic band of benzamide and cholesterol can be identified from their FTIR spectra. Moreover, the FTIR spectrum of the separated benzamide and cholesterol spots on the narrowband TLC plate are roughly the same as the reference IR spectra of pure benzamide and cholesterol.
The present work demonstrates that TLC/FTIR technique is applicable in analysis of complex mixtures.

\section{Conflict of Interests}

The authors declare that there is no conflict of interests regarding the publication of this paper.

\section{Acknowledgment}

Financial support from the National Natural Science Foundation of China (51373003) is gratefully acknowledged.

\section{References}

[1] M. Srivastava, Ed., High-Performance Thin-Layer Chromatography (HPTLC), Springer, Heidelberg, Germany, 2011.

[2] L. Y. He, Ed., Method and Application of Planar Chromatography, Chemical Industry Press, Beijing, China, 2005.

[3] J. Sherma, "Planar chromatography," Analytical Chemistry, vol. 82, no. 12, pp. 4895-4910, 2010.

[4] J. G. Wu, Ed., Modern FTIR Spectroscopy Technology and Application, Science and Technology Press, Beijing, China, 1994.

[5] S. F. Weng, Ed., Fourier Transform Infrared Spectrometer, Chemical Industry Press, Beijing, 2nd edition, 2010.

[6] R. N. McCoy and E. C. Fiebig, "Technique for obtaining infrared spectra of microgram amounts of compounds separated by thin layer chromatography," Analytical Chemistry, vol. 37, pp. 593595, 1965.

[7] P. A. Sturm, "Quantitative determination of individual tocopherols by thin layer chromatographic separation and spectrophotometry," Analytical Chemistry, vol. 38, no. 9, pp. 12441247, 1966.

[8] C. J. Percival and P. R. Griffiths, "Direct measurement of the infrared spectra of compounds separated by thin-layer chromatography," Analytical Chemistry, vol. 47, no. 1, pp. 154$156,1975$.

[9] M. P. Fuller and P. R. Griffiths, "Diffuse reflectance measurements by infrared fourier transform spectrometry," Analytical Chemistry, vol. 50, no. 13, pp. 1906-1910, 1978.

[10] M. P. Fuller and P. R. Griffiths, "Infrared microsampling by diffuse reflectance Fourier transform spectrometry," Applied Spectroscopy, vol. 34, pp. 533-539, 1980.

[11] G. E. Zuber, R. J. Warren, P. P. Begosh, and E. L. O’Donnell, "Direct analysis of thin-layer chromatography spots by diffuse reflectance fourier transform infrared spectrometry," Analytical Chemistry, vol. 56, no. 14, pp. 2935-2939, 1984.

[12] L. B. Lloyd, R. C. Yeates, and E. M. Eyring, "Fourier transform infrared photoacoustic spectroscopy in thin-layer chromatography," Analytical Chemistry, vol. 54, no. 3, pp. 549-552, 1982.

[13] R. L. White, "Analysis of thin-layer chromatographic adsorbates by Fourier transform infrared photoacoustic spectroscopy," Analytical Chemistry, vol. 57, no. 9, pp. 1819-1822, 1985.

[14] N. D. Danielson, J. E. Katon, S. P. Bouffard, and Z. Zhu, "Zirconium oxide stationary phase for thin-layer chromatography with diffuse reflectance fourier transform infrared detection," Analytical Chemistry, vol. 64, no. 18, pp. 2183-2186, 1992. 
[15] S. P. Bouffard, J. E. Katon, A. J. Sommer, and N. D. Danlelson, "Development of microchannel thin-layer chromatography with infrared microspectroscopic detection," Analytical Chemistry, vol. 66, no. 13, pp. 1937-1940, 1994.

[16] W. He, R. Shanks, and G. Amarasinghe, "Analysis of additives in polymers by thin-layer chromatography coupled with Fourier transform-infrared microscopy," Vibrational Spectroscopy, vol. 30, no. 2, pp. 147-156, 2002.

[17] W. He, G. Cheng, F. Zao, Y. Lin, J. Huang, and R. Shanks, "Separation and identification of multicomponent mixture by thin-layer chromatography coupled with Fourier transforminfrared microscopy," Spectrochimica Acta Part A, vol. 61, no. 8, pp. 1965-1970, 2005.

[18] X. Liu, Q.-H. Pan, J. Ding et al., "Using barium fluoride fine particles as stationary phase for TLC/FTIR analysis," Spectroscopy and Spectral Analysis, vol. 31, no. 7, pp. 1767-1771, 2011.

[19] Q. Zhu, X. Su, H. J. Wu et al., "The analysis for silver iodide fine particles of TLC/FTIR matrix," Spectroscopy and Spectral Analysis, vol. 32, no. 7, pp. 1790-1794, 2012.

[20] W. Liu, H. J. Wu, X. P. Wang et al., “ Preparation and application of barium fluoride particles as stationary phase for TLC-FTIR analysis," Chemical Journal of Chinese Universities, vol. 34, no. 6, pp. 1347-1352, 2013.

[21] F. Wang and H. J. Wu, "Development of narrow-band TLC plates for TLC/FTIR analysis," Analytical Methods, vol. 5, pp. 4138-4144, 2013. 

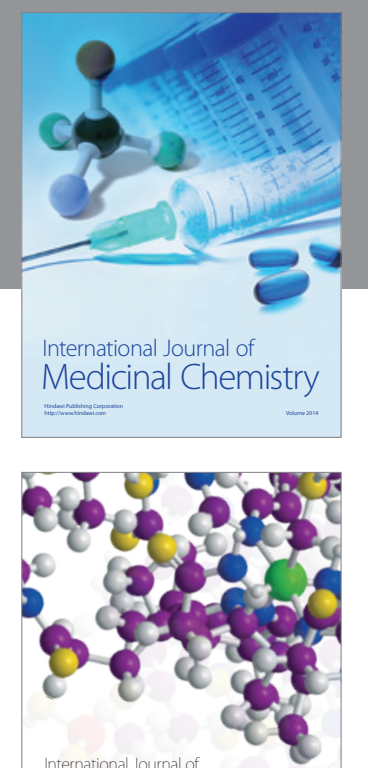

\section{Carbohydrate} Chemistry

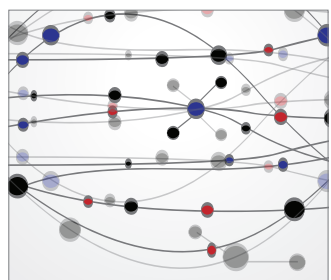

The Scientific World Journal
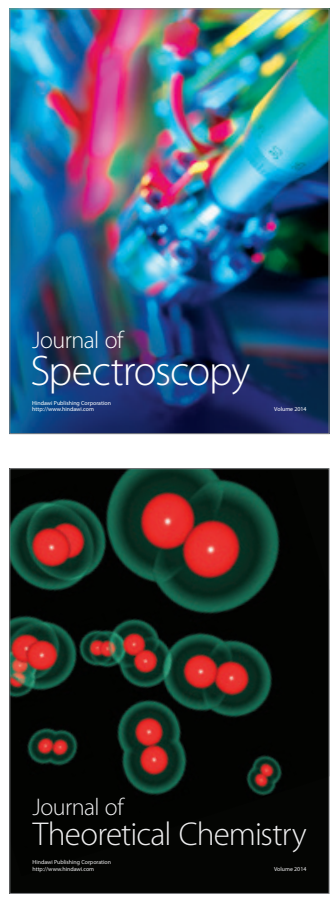
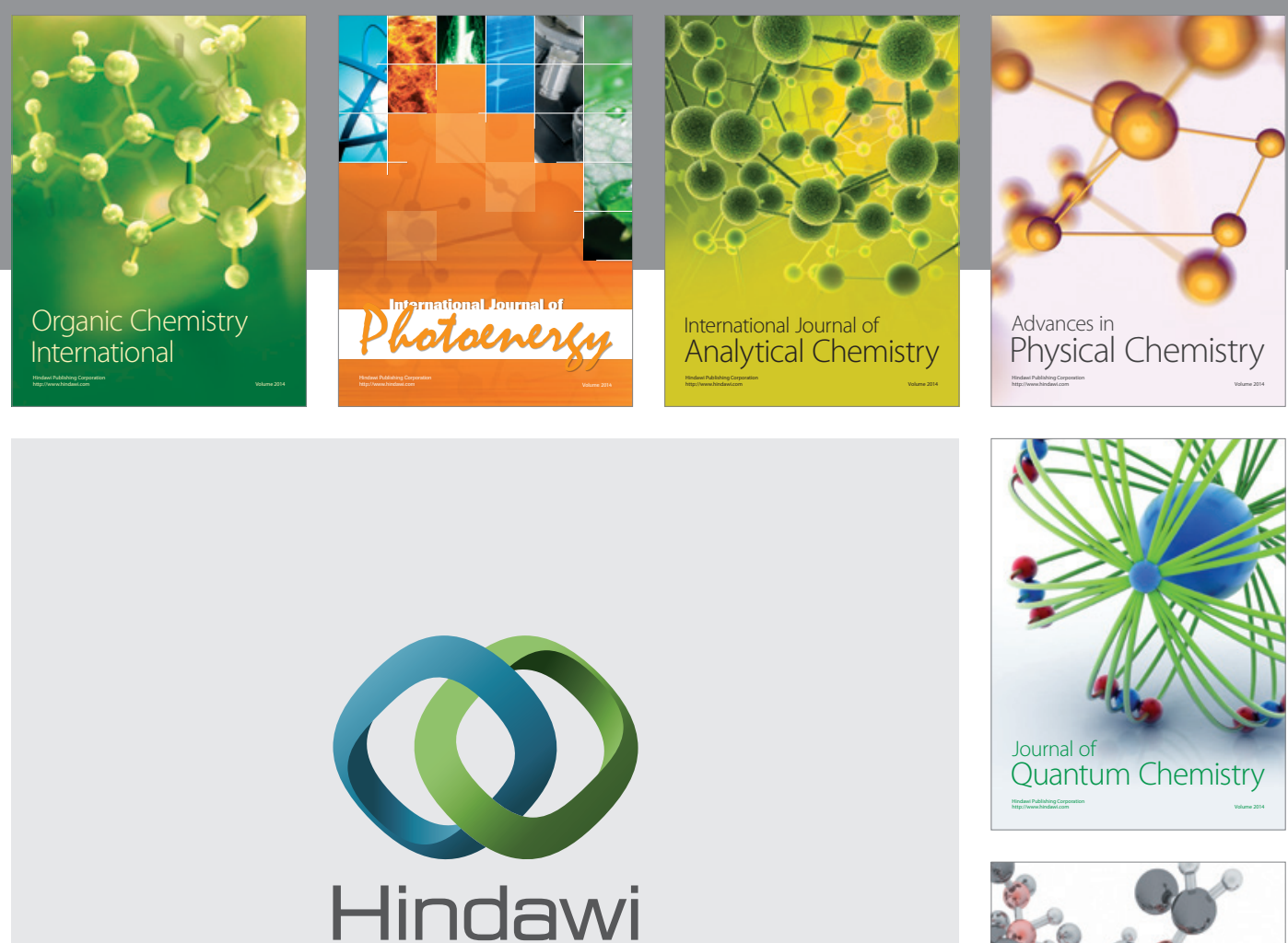

Submit your manuscripts at

http://www.hindawi.com

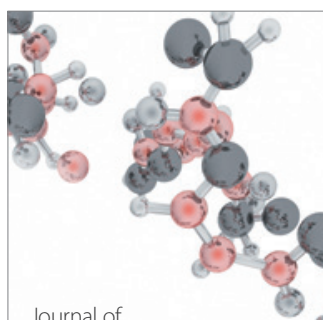

Analytical Methods

in Chemistry

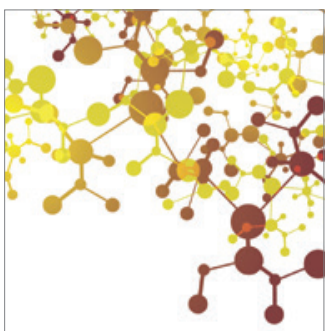

Journal of

Applied Chemistry

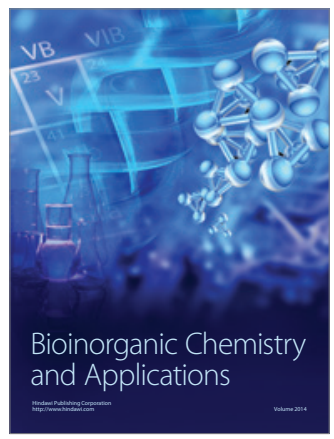

Inorganic Chemistry
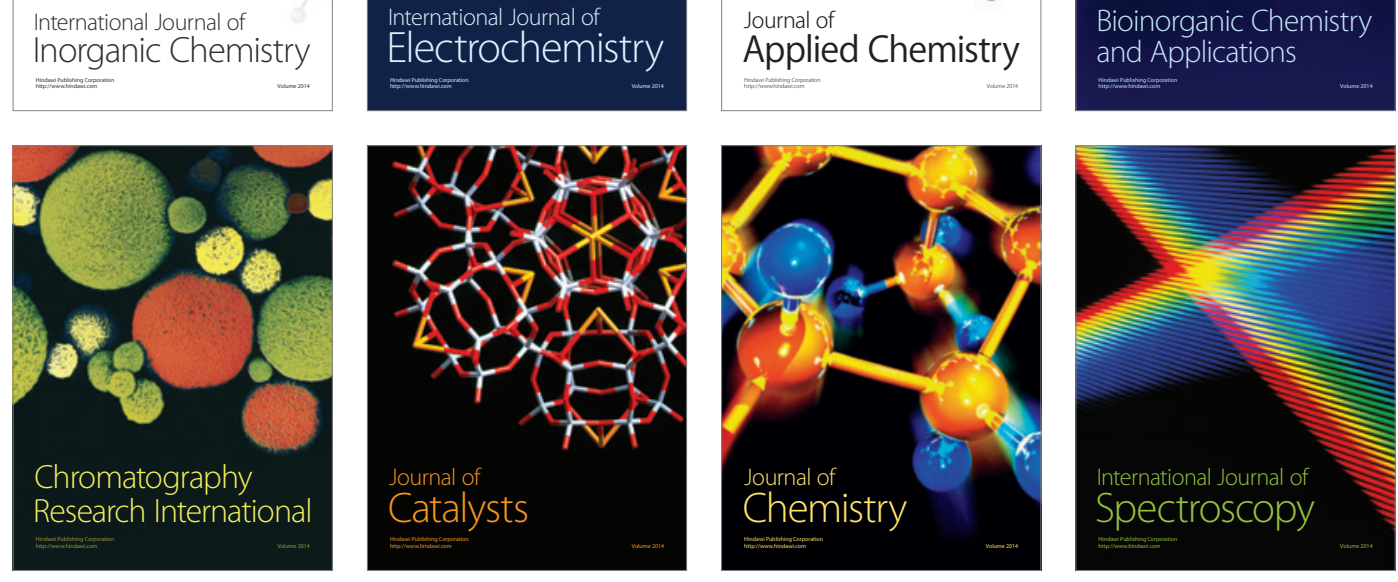